\title{
A gate hit detection system for canoe slalom
}

\author{
John Ringwood \\ Dept. of Electronic Engineering \\ Maynooth University, Ireland \\ E-mail: john.ringwood@eeng.nuim.ie
}

\author{
Wenkan Qian \\ Dept. of Electronic Engineering \\ Maynooth University, Ireland
}

\author{
Javier Conde Fernandez \\ Ernst and Young \\ Plaza Pablo Ruiz Picasso, Madrid, Spain \\ E-mail: javier.condefernandez@es.ey.com
}

\begin{abstract}
Many sports now look to technology to resolve contentious decisions, for example in tennis, Gaelic games, snooker, association football, etc. However, the sport of canoe slalom, an Olympic sport, still relies completely on the subjective decision of a human judge, as to whether a competitor has touched a 'gate' or not. With the time difference between gold and bronze medals frequently being less than 2 secs, a single 2 second penalty has a significant impact and reliable, objective and repeatable judging is vital. This paper reports on a prototype slalom pole hitdetection system, which has been developed in consultation with the International Canoe Federation (ICF) Slalom Committee. Index Terms-Canoe, kayak, slalom, penalty, classification
\end{abstract}

\section{INTRODUCTION}

Over the past decade or two, there has been a proliferation of technology-assisted judging in a wide variety of sports. While video reply has played a part in many sports for some considerable time (e.g. athletics, rugby, etc), other technology (including post-processing of video, or a fusion of video images) has been less prevalent, with fencing being one of the exceptions, with initial experimentation of electronic scoring dating back to 1896 [1]. Possibly one of the best known of the recent developments is the Hawkeye system for tennis [2], with other similar applications including goal-line technology for association football [3] and Gaelic games, leg-beforewicket (LBW) decisions in cricket [4], etc. Other related developments include virtual spectator technology [5], used in a variety of sports including yacht racing, which could also have application in rule judging.

Most technology-assisted judging systems are used in an advisory capacity, rather than in real time. The reasons for this are multi-faceted:

- The technology may not be capable of real-time computation,

- There is no need for a real-time indication, or

- The sport (participants and organisers alike) may wish to retain traditional human aspects of the sport.

For example, the Hawkeye system has a computational delay of approximately $5 \mathrm{~s}$, rendering real-time implementation impossible. However, simpler systems, such as hit detection in fencing, are both feasible and employed in real time.

Canoe sport began in the late 1800's in North America, with races between the native Indians and early colonists [6]. In the slalom discipline of canoe-kayak sport, where competitors are required to negotiate a number of slalom 'gates' on a whitewater course, the first world championships were held

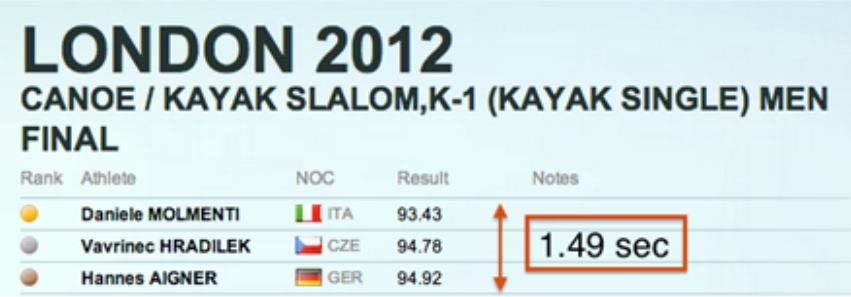

Fig. 1. Podium position for men's kayak slalom at London 2012 Olympics

in 1949, and appeared initially in the Olympic games in 1972. Following a hiatus until 2002, slalom canoeing (kneeling, using a single paddle) and kayaking (sitting, using a double paddle) has since been an integral part of all Olympic games. Briefly, the rules [7] of canoe/kayak slalom (hereafter referred to as 'slalom' for brevity) require competitors to negotiate a course of between 18 and 25 gates consisting of vertical poles between which competitors must pass. At least 6 gates must be negotiated upstream (red/white poles) with the remainder downstream (green/white) and gates must be negotiated in strict order. Typically, courses measure between $200 \mathrm{~m}$ and $400 \mathrm{~m}$ from start to finish, with a transit time of around 100 s. Time penalties are incurred as follows:

$2 \mathrm{~s}$ if one, or both poles, of a gate is 'touched', and

$50 \mathrm{~s}$ if a gate is missed.

No further clarification is provided on the precise meaning of 'touched', with some contact being obvious and resulting in pole displacement, while other more subtle contact may result in pole rotation only, or no perceptible movement at all. A touch penalty is awarded if any part of the competitor's canoe or kayak, body or equipment (including paddles, helmet, buoyancy aid, etc). However, rule 29.8 states that "At all times, the benefit of any doubt must be given to the competitor". Currently, at major competitions (Olympics, World Championships, World Cups) penalties are judged on the basis of an individual human judge for each gate, with the possibility for video review following, for example, a protest by the competitor or competitor's team. Difficulties experienced in the current system include:

1) The natural displacement of gate poles due to water splashes (recall that a whitewater course is used) and wind,

2) The lack of having a $360^{\circ}$ perspective on each pole, either by the judge or video system (note that a set of 6- 
10 video cameras are used to cover the complete course),

3) Any inconsistency in human judging, either between judges, or between individual competitors with respect to a single judge, and

4) The lack of a scientific definition of a 'touch', and its precise measurement.

The consequences of a touch, or a mis-judged touch are significant. Fig.1 shows the podium results from the Men's Slalom K1 (individual kayak) at the London 2012 Olympics. Note that the gold to bronze medal positions are separated by less than a single touch penalty $(2 \mathrm{~s})$ !

In this paper, we present a prototype electronic system for detecting touches on a slalom pole. The system is based on an inertial measurement unit, with an Arduino microcontroller used to capture the data and transmit the data via Bluetooth to a PC, where data pre-processing takes place and a classifier implemented to distinguish between normal movement of the pole due to water and wind disturbances, if present, and pole touches by competitors. To the best of the authors' knowledge, it is the first operational prototype system to have been developed, though a design for such a system was considered in [8].

The overall configuration of the pole hit classification system is shown in Fig.2. Serial data from the bluetooth receiver is packaged into $10 \mathrm{~s}$ frames, with both frequency-domain and time-domain inputs being used to feed a data reduction stage (using principal components analysis (PCA)). The PCA outputs then feed the classifier which produced an output in the range $0 \rightarrow 1$, with a ' 1 ' indicating a hit.

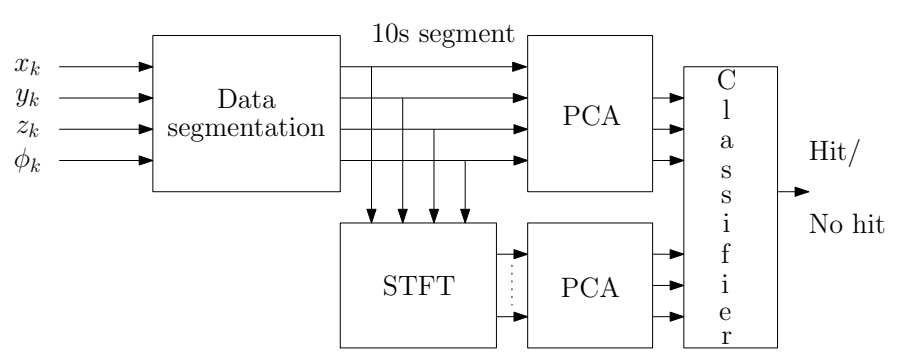

Fig. 2. Overview of pole hit classification system

The remainder of the paper is organised as follows: The hardware setup, including the sensor system, data logging and data transmission, is considered in Section II, while the test setup and data logged are reported in Section III. Sections IV and $\mathrm{V}$ describe the design of the data preprocessing algorithms and classifier, respectively, with results and conclusions documented in Sections VI and VII respectively.

\section{HARDWARE SETUP}

This section documents the components used to make the measurements on the pole, assemble the data for transmission and transmit the data to a PC for further processing.

\section{A. Sensor selection}

A variety of potential sensors were considered for this application, including aural, electrical contact, mechanical, optical (infra red (IR) and video) and motion. Although audio detection has found application in cricket [9] is was deemed inappropriate for the noisy environment of slalom, while an electrical contact sensor system would have potential difficulties with moisture and require poles to be made of specialised (expensive) material. Mechanical and IR sensors were discounted in [8] for various reasons and while visionbased systems have good potential, they were deemed to be too expensive due to the requirement for a camera per pole, with at least 36 poles required to be instrumented. In addition, it is unlikely that a vision-based system could operate in realtime and data transmission requirements are also likely to increase the data handling hardware costs. Motion sensors,

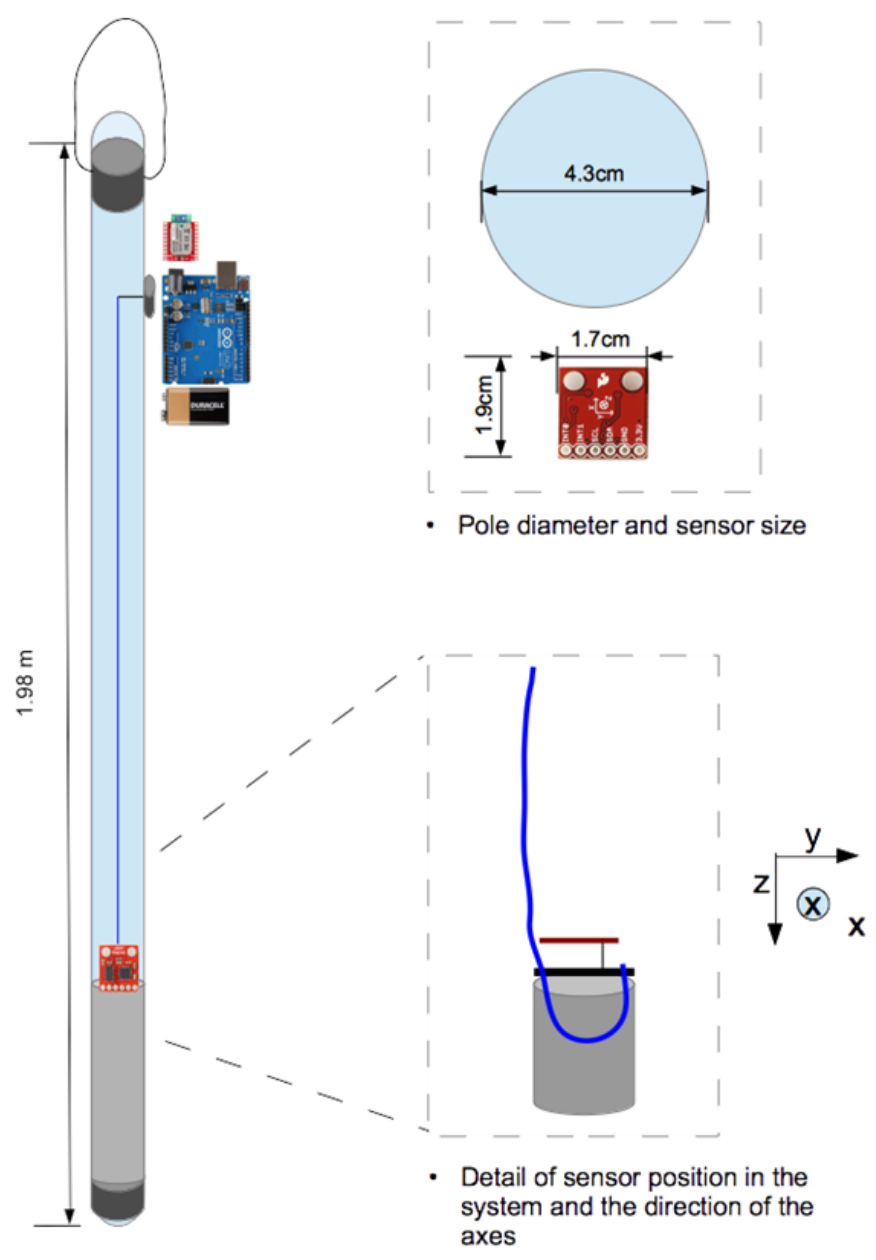

Fig. 3. Overall setup of instrumented slalom pole

including both accelerometer and gyroscopic systems, present a potentially useful and cost-effective solution and require no specialised pole materials, with the possibility to retro-fit existing slalom poles. Typical inertial measurement units (IMUs) contain both accelerometer and gyroscopic measurements and often also include magnetometers. An IMU Digital Combo Board was chosen, which provides 6 degrees of freedom (DoF) and contains the ITG3205/ADXL345 gyroscope/accelerometer devices. All three translations DoFs were employed, along 


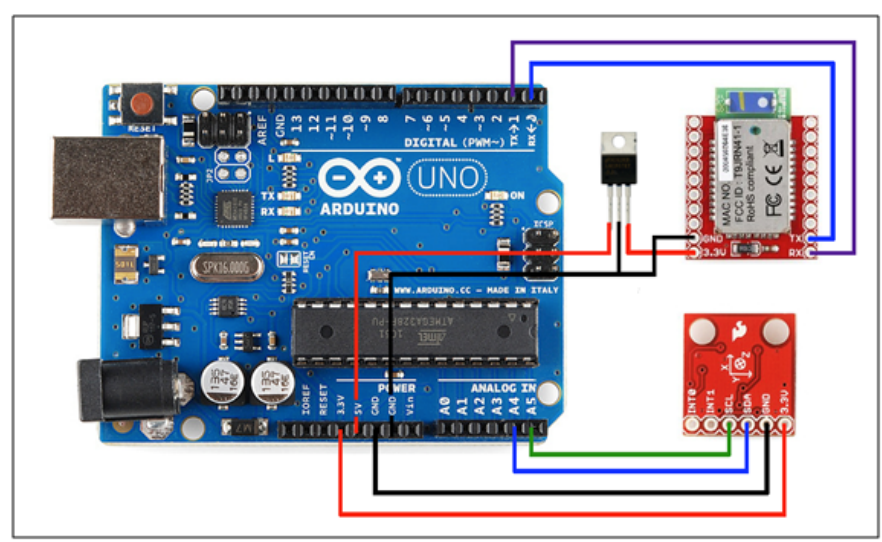

Fig. 4. Arduino board with Bluetooth and IMU connections

with rotation around the vertical axis (yaw). This sensor system offers $2 \mathrm{~g}$ full range sensitivity for translational DoFs and $2000 \%$ for rotation. The IMU is positioned towards the bottom of the pole (poles are hung from the top) to maximise sensitivity, as shown in Fig.3.

\section{B. Data communication}

A standard Arduino Uno board was interfaced to the IMU, via the $I^{2} C$ digital interface, which facilitates serial signal and clock data transmission. The system is set up to allow the Arduino to operate as a master, with the gyroscope and accelerometer as slave units. The Arduino can therefore set the required sensitivity level in the accelerometer $( \pm 2 g, \pm 4 g, \pm 8 g, \pm 16 g)$ and read the measured linear and angular acceleration from the appropriate memory locations on the IMU. A RN41XVC-I/RM Class 1 Bluetooth module is chosen for data transmission, with a range of $100 \mathrm{~m}$ (note that Bluetooth Class 2 provides insufficient range for this application, at $10 \mathrm{~m}$ ). The data transmission rate used is 9600 bps, providing a good compromise between transmission rate and channel integrity. Power for the system is supplied via a $9 \mathrm{~V}$ battery, providing power directly to the Arduino board which, in turn, supplies the IMU with $3.3 \mathrm{~V}$. A separate regulator, using the $5 \mathrm{~V}$ output on the Arduino board, is used to supply the Bluetooth module with 3.3 V. The data transmission electronics are shown in Fig.4.

\section{The test slalom pole}

The pole employed for testing is a hollow plastic cylinder, allowing sufficient space for insertion of the IMU and data transmission electronics. ICF rules, which govern the type of gate pole which may be used, specify a pole height of between $1.6 \mathrm{~m}$ and $2 \mathrm{~m}$ and an outside diameter of between $3.5 \mathrm{~cm}$ and $5 \mathrm{~cm}$. No exact specification is given for the weight, with the exception of the guideline "of sufficient weight that motion caused by wind is not excessive" and, typically, poles employed for most international competitions weigh approximately $1.3 \mathrm{~kg}$. The weight of the bare test pole was $820 \mathrm{~g}$ and, complete with instrumentation, came in at $1.1 \mathrm{~kg}$, which is deemed to be acceptable. The overall configuration of the instrumented slalom pole is shown in Fig.3. A plastic cover was fixed to the top of the pole to prevent water ingress.

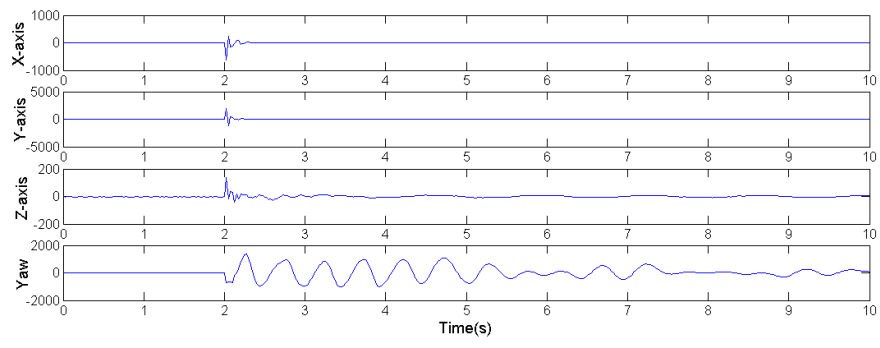

Fig. 5. IMU recordings corresponding to a pole hit at $2 \mathrm{~s}$

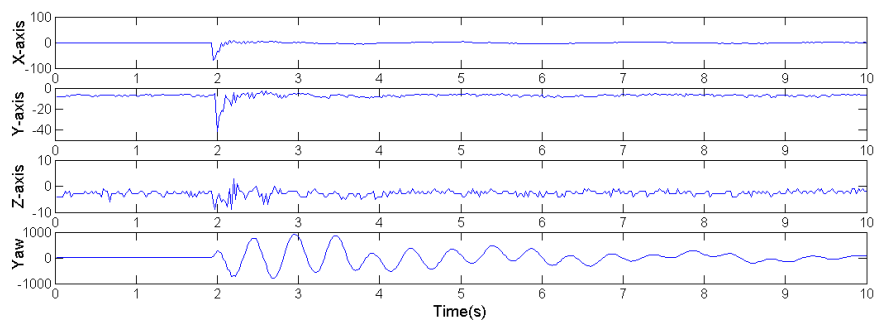

Fig. 6. IMU recordings corresponding to a water splash disturbance at $2 \mathrm{~s}$

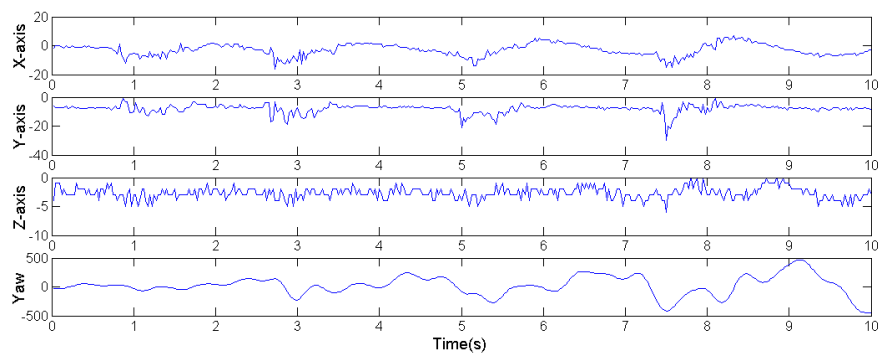

Fig. 7. IMU recordings corresponding to wind disturbances

\section{Test DATA Logging}

Both lab-based and water-based testing was carried out to $\log$ suitable data, containing both disturbances (water and wind) and various types of hits on the pole from body, boat and paddle. Fig. 8 shows the water-based testing setup, where the white pole is the active pole while, by way of example, Figs.5, 6 and 7 show the time records for a hit, and water and wind disturbances respectively, which give an indication of the challenge facing the classifier in discriminating between pole hits and ambient disturbances. Fig.9 shows a typical segment of training data with a mixture of disturbances and hits. For the statistical classifier results, a total of $135 \mathrm{~s}$ of training and 105 $\mathrm{s}$ of test data was recorded. The full training data contained a total of 9 specific disturbances and 17 hits, while the test data contained a total of 5 specific disturbances and 17 hits. 


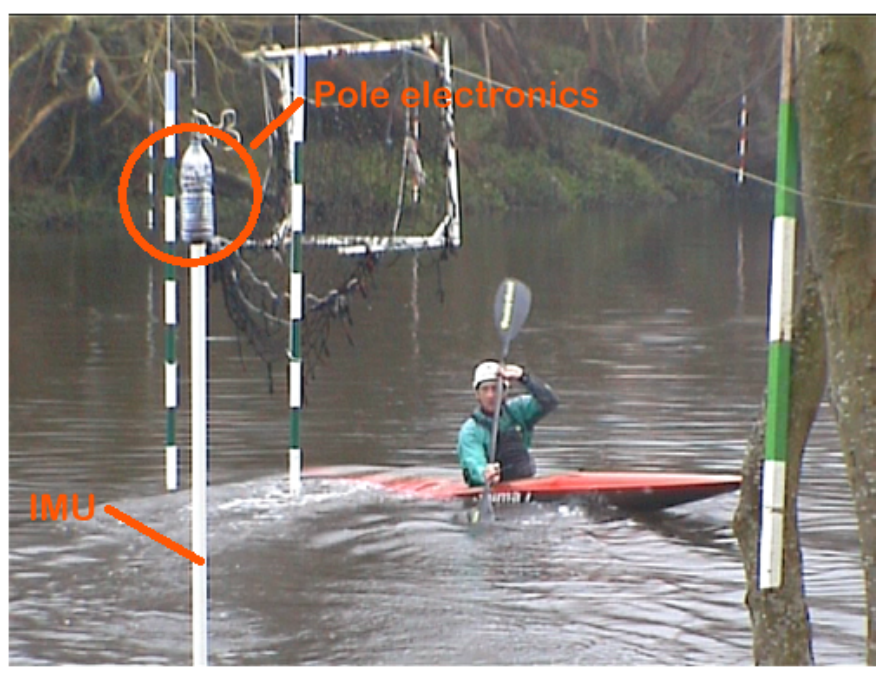

Fig. 8. River testing for data collection

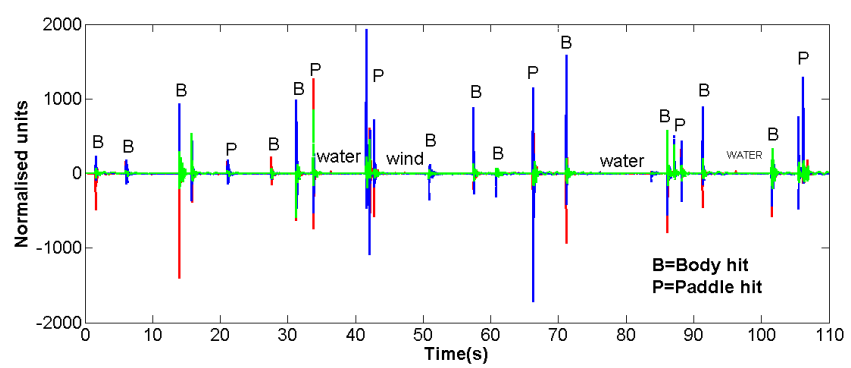

Fig. 9. Typical training data segment

\section{DATA PREPROCESSING}

The raw IMU data is pre-processed in order to provide a set of suitable variables to the classifier in an appropriate format to make the job of the classifier easier and to facilitate interpretation. As a first step, the data is segmented into 10s frames, which is regarded as the time that a single gate is 'active'. The choice of $10 \mathrm{~s}$ is based on an average inter-gate transit time of 3.9s (from the London Olympics) with average mimimum and maximum inter-gate transit time (across different competitors) being $1.1 \mathrm{~s}$ and $9.8 \mathrm{~s}$, respectively. The point at which a gate becomes 'active' would be specified by the supervisory coursewide software, which is not considered in this study.

\section{A. Data transformation}

In terms of candidate data transformations, a frequency transformation is perhaps an obvious candidate, giving the potential to distinguish between the relatively high frequencies of impact hits and the relatively low frequencies of softer motion due to water splashes or wind excitation. However, some time localisation within the 10 s window is important, so the discrete-time short-term Fourier transform (STFT) is employed:

$$
X(m, \lambda)=\sum_{n=-\infty}^{\infty} x[n] w[n-m] e^{-j \lambda n}
$$

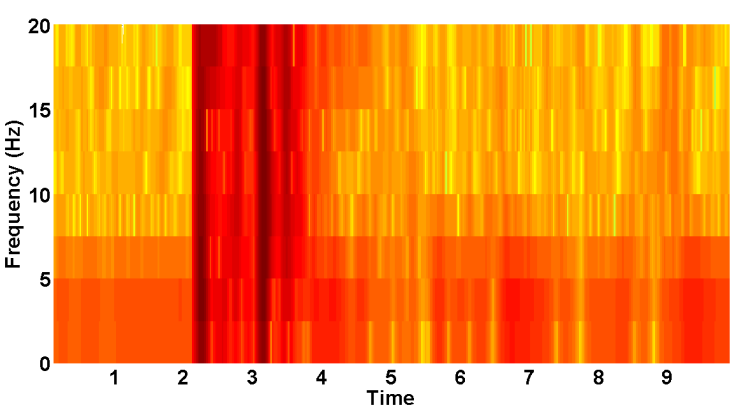

Fig. 10. STFT for an arm hit at 2 seconds within a 10 segment

where $n$ is the time index, $\lambda$ the frequency index and $w$ the windowing function employed. For this study, a Hanning [10] window function was employed, with a window length of 10 samples and an overlap of 9 samples. By way of example, Fig.10 shows the STFT for an arm hit at $2 \mathrm{~s}$ within a $10 \mathrm{~s}$ segment. While the frequency discretization is relatively coarse for the STFT parameters chosen, the time localisation of the hit is adequate for the application.

For the STFT, and the corresponding Hanning window length of 10 samples, with an overlap factor of 9/10 (i.e. 9 samples), the original 400 samples per frame, for each of the 4 IMU signals, converts to 8 spectral points every sample (10 datapoints, zero padded out to 16 for FFT use and taking the first $1 / 2$ of the spectrum). However, a small number of samples ( 9 in total) are lost at the start and end of the data record due to the windowing, reducing the effective 10s data record by $0.225 \mathrm{~s}$. The choice of a $9 / 10$ overlap gives a good compromise between the time localisation and frequency resolution. The time-domain signals fed to the next stage of preprocessing are appropriately subsampled, in order to be commensurate in time position and length with the frequencydomain signals. Considering the 4 separate IMU channels, a total of 32 frequency-domain signals can now be provided to the classifier.

The resulting STFT data, with 8 frequency datapoints per IMU channel at each time step, are now considered in addition to the original 4 time domain IMU signals as inputs to the classifier.

\section{B. Data dimension reduction}

Considering both time- and frequency-domain measurements, a total of $12(=4+32)$ signals are now available to the classifier. In order to eliminate significant levels of linear dependence between signals, principal component analysis (PCA) [11] is separately applied to the sets of time- and frequency-domain signals, as shown in Fig.2. Fig.12 shows the variance explained for the frequency-domain principal components, while Fig.11 shows the variance explained for the time-domain principal components. Overall, 3 (from 32) components, representing $98.3=(62.5+32.5+3.3) \%$ of the variance explained, are selected for the frequency-domain signals, while 2 (from 4) components, representing $97.8 \%$ 


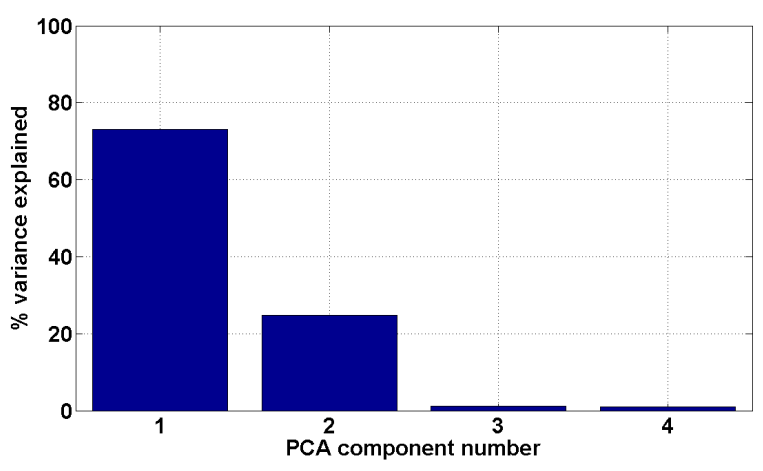

Fig. 11. Variance explained for time-domain PCA components

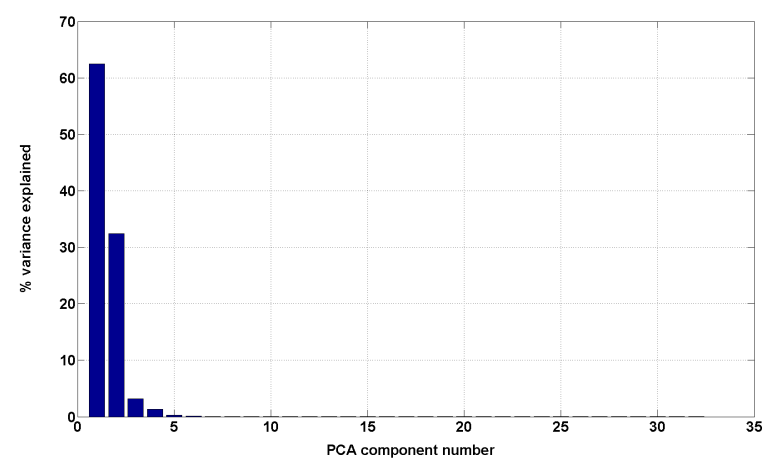

Fig. 12. Variance explained for frequency-domain PCA components

of the variance explained, are selected for the time-domain signals.

\section{Classifier Design}

The classifier employed is a multi-layer perceptron (MLP) artificial neural network (ANN). The inputs to the network are the principal component scores for time- and frequencydomain signals, with a total number of classifier inputs of 5 , as documented in SectionIV-B. A 3-layer ANN is employed, utilising tan-sigmoid neurons (since the input signals are bipolar) with a log-sigmoid output neuron, since we denote a hit with a ' 1 ' and no hit with a ' 0 '. 3 neurons are employed in the first layer, with 5 neurons in the second layer and a single output neuron. The network is trained using a scaled conjugate gradient (backpropagation) algorithm [12] using a variety of initial conditions to ensure a good local performance surface minimum is achieved. With the range of the log-sigmoid output neuron in the range $0 \rightarrow 1$, any number between the extremities can be loosely interpreted as the probability of a hit.

5,400 samples of the recorded IMU signals are used for training data, incorporating 26 events, comprising 9 disturbance and 17 hit events. The target signal is specified as follows. All non-hit frame target sequences are set to zeros. For training data frames containing a hit, the segment of the training data where all the magnitude of the original $(x, y, z, \phi)$ time-domain signals from the IMU exceed 100 are

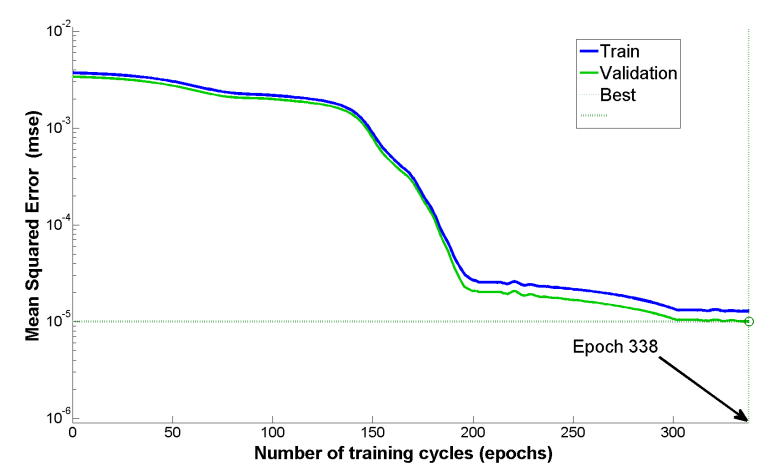

Fig. 13. Training record for the ANN classifier
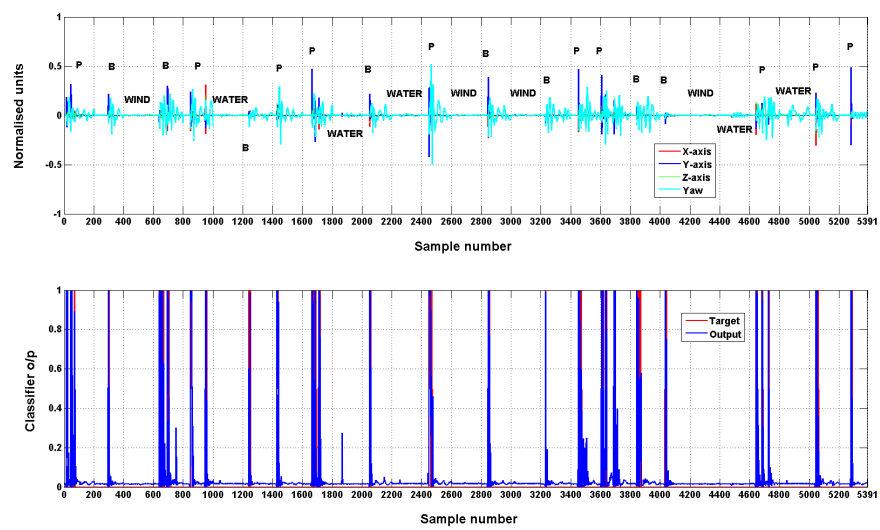

Fig. 14. Classifier performance on training data

assigned a target of 1 . Note that the full range for each of the IMU signals is 4096 , by virtue of 12-bit binary representation. Therefore, 100/4096 represents roughly $1 / 40$ of the full range.

The training record of network is shown in Fig.13. Early stopping was employed, based on the classifier mean squared error (MSE) measured on validation data randomly extracted (at a $15 \%$ level) from the original training data. This ensures that the ANN classifier retains the capability to generalise well to unseen data. The performance of the classifier on the training data is shown in Fig.14.

\section{REsults}

Unseen test data, comprising 4,400 time samples containing 22 events, containing 5 disturbance and 17 hit events, were used to test the hit classification system. Using a threshold of 0.5 applied to the classifier output, all test hits were classified correctly, with no false positives. The classifier output threshold can be adjusted for a particular site to achieve an optimum balance between sensitivity and false positives. Fig. 15 shows the variation in the IMU $(x, y, y)$ signals (with events labelled) and the corresponding classifier output, while Fig.16 shows the variation in time- and frequency-domain test scores on the test data. It can be noted that the classifier saturates at the target points and the scale of the water and wind disturbances are relatively modest in amplitude compared to the hit signals. 

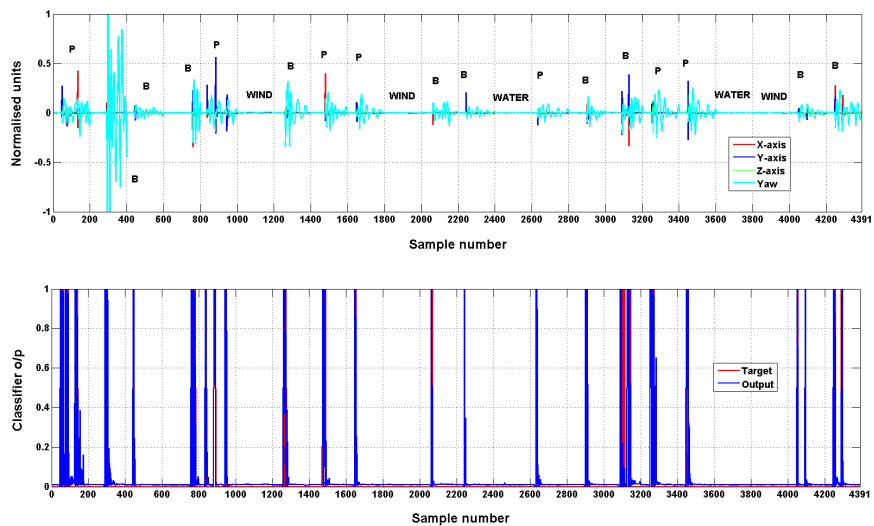

Fig. 15. Classifier performance on unseen test data
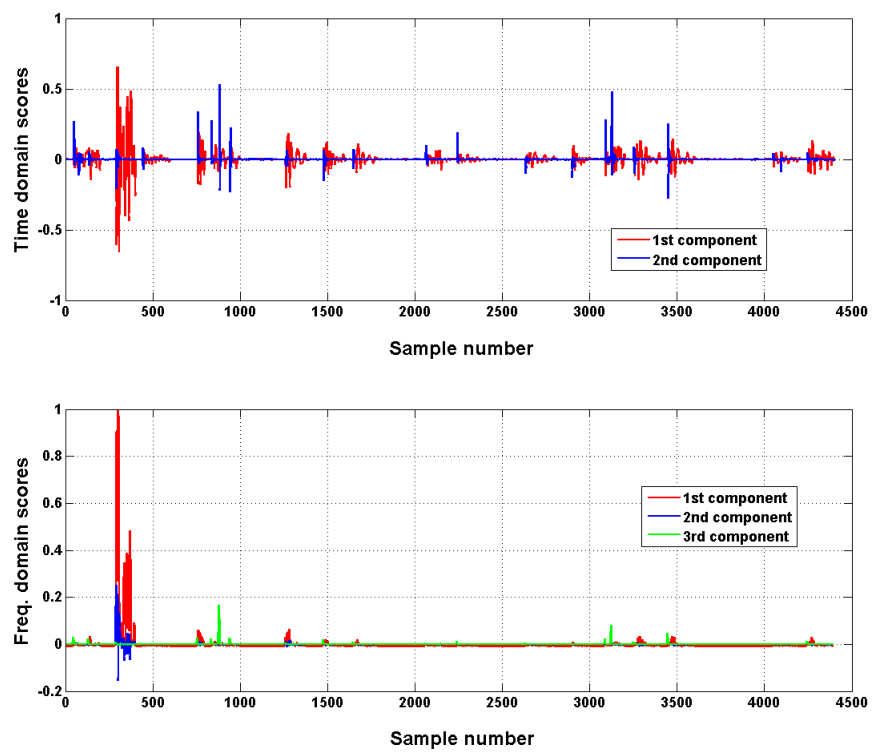

Fig. 16. Classifier performance on unseen test data

There may be a natural amplitude limitation (in acceleration) on the scale of the disturbances, but this would need to be confirmed by further testing.

\section{CONCLUSIONS}

A hit classification system for canoe/kayak slalom has been designed, utilising an IMU as a primary sensor, with data transmitted wirelessly to a central station for hit classification. The classifier employs frequency-domain transformation and PCA is applied to both time- and frequency-domain signals to provide a set of suitable signals for the classifier to discriminate between hits and other IMU disturbances due to to wind and water splashes. For the data considered, the system works well for a wide range of classifier thresholds, suggesting that there is considerable margin for successful operation with more challenging data. To this end, more and varied test data is required to assess the operation of the system at a range of sites and the system is shortly due to be evaluated by the
Olympiastützpunkt Bayern at the Eiskanal (former Olympic slalom site) in Augsburg.

While it is impossible, given the limitations of both the IMU and the classifier, to have infinite sensitivity to hits and zero sensitivity to disturbances, the system provides an acceptable level of sensitivity and, most importantly, is entirely objective and consistent in hit classification. This represents a major advantage of the system over current human (subjective) judging and should appeal to elite competitors and organisers alike. The system is computationally simple and can be implemented with a latency of about $0.1 \mathrm{~s}$, making it suitable for 'real time' (e.g. green/red visual) display using an indicator on the poles themselves. This could be achieved using the Bluetooth connection from the central classifier system to the pole.

Finally, the system is inexpensive at approximately $€ 50$ per pole (assuming some custom design) and is therefore suitable for deployment to a complete 25 -gate ( 50 pole) course. However, for full course operation, some further wireless data transmission issues need to be addressed.

\section{ACKNOWLEDGMENT}

The authors are grateful for the contributions of Dipl.Ing. Thomas Schmidt, ICF Slalom Committee (and former Olympic kayak slalom champion), Prof. Dr.-Ing. Franz Raps of Hochschule Augsburg and John Maloco of the Dept. of Electronic Engineering, Maynooth University to this project.

\section{REFERENCES}

[1] R. Crosnier and K. Gibson, Fencing with the Electric Foil: Introduction and Tactics. Faber \& Faber, 1961.

[2] N. Owens, C. Harris, and C. Stennett, "Hawk-eye tennis system," in IEE conference publication. Institution of Electrical Engineers, 2003, pp. $182-185$.

[3] N. Ancona, C. Cicirelli, A. Branca, and A. Distante, "Goal detection in football by using support vector machines for classification," in Neural Networks, 2001. Proceedings. IJCNN'01. International Joint Conference on, vol. 1. IEEE, 2001, pp. 611-616.

[4] H. Collins and R. Evans, "Sport-decision aids and the csi-effect: Why cricket uses hawk-eye well and tennis uses it badly," Public Understanding of Science, vol. 21, no. 8, pp. 904-921, 2012.

[5] L. Zentai and A. Guszlev, "Spatial tracking in sport," in Location based services and TeleCartography. Springer, 2007, pp. 593-605.

[6] N. Howell and M. L. Howell, Sports and games in Canadian life, 1700 to the present. Macmillan of Canada, 1969, no. 29.

[7] I. C. Federation, "Icf canoe slalom competition rules," International Canoe Federation, Tech. Rep., 2015.

[8] K. Lockwood, C. Munshaw, J. So, and A. Penna, "Design specification for slalom race organiser," School of Engineering Science, Simon Fraser University, Tech. Rep., 2007.

[9] R. Rock, A. Als, P. Gibbs, and C. Hunte, "The 5 th umpire: Automating crickets edge detection system," Journal of Education, Informatics \& Cybernetics, vol. 11, no. 1, 2013.

[10] R. Blackman and J. Tukey, The Measurement of Power Spectra. Dover: New York, 1958.

[11] G. H. Dunteman, Principal components analysis. Sage, 1989, no. 69

[12] M. F. Møller, "A scaled conjugate gradient algorithm for fast supervised learning," Neural Networks, vol. 6, no. 4, pp. 525-533, 1993. 